(i)

\title{
Editorial
}

\section{Sexual and Reproductive Health Issues in Women Living with HIV/AIDS, Epilepsy and Gynaecological Cancers}

As per WHO, sexual health is a state of complete physical, emotional, mental and social wellbeing in relation to sexuality; it is not merely the absence of disease, dysfunction or infirmity. Sexual and reproductive health requires a positive and respectful approach to sexuality and sexual relationship, as well as the possibility of having a pleasurable and safe sexual experiences, free of coercion, discrimination and violence. Comprehensive sexual and reproductive health programmes and services are the most effective approaches for preventing women mortality, morbidity, fighting HIV and AIDS, preventing and treating gynaecological cancers and meeting the sexual and reproductive health needs of women and young people while promoting human rights.

Healthy women need health care in order to be able to carry their sexual/reproductive function, and to carry them safely and successfully. During the last fifty years, there has been a vast expansion of health technologies and of health services to provide women with certain elements of reproductive health care. These services were not, however, without shortcomings. Apart from inadequate allocation of resources, the major shortcomings were in the philosophy with which services were provided. Women were considered as means in the process of reproduction and as targets in the process of fertility control. The services were not provided to women as ends in themselves. Women were benefitted but were not there at the center of the process. The needs of women have been traditionally addressed within the concept of maternal and child health $(\mathrm{MCH})$. The needs of the women were, however, submerged in the needs of the mother. MCH services tend to focus on the healthy child as the successful outcome. While mother's care is very much needed for this successful outcome because of the investment she make in the process of reproduction, this focus resulted in less emphasis being put on caring for the health risk to which mothers are liable during pregnancy and childbirth, and putting in place the essential obstetric functions and facilities to deal with them. As a result, the tragedy of maternal mortality in developing countries has now reached dimensions that cannot be ignored.

With the mindset that the women are means and not the ends, important health needs in the reproductive process have been left unmet. For example, with all its benefits for the quality of life, the family planning programme has left women with some genuine concerns as well as unmet needs. Contraceptives are meant to be used by women to empower themselves by enhancing their choices, and controlling their fertility, their 
sexuality, their health and thus their lives. Family planning, however, can be used and has been used by the governments and others to control birth rather than to empower women. The family planning programme has been largely demographic driven. As far as policy makers are concerned, women were often considered as objects and not subjects. Some governments are short-sighted; not to see that when women are given real choice, and the information and means to implement their choice, they will make the most rational decision themselves, for their communities and ultimately for the world at large.

Infertility may not be a serious hazard as far as physical health is concerned, but can be a major cause of mental and social ill-health. It is not fare that society should provide care to the fertile and reproductive women, but should neglect the suffering of those who are unable to conceive due to infertility or suffering with epilepsy or situations where antiepileptic drug (AED) therapy has made the pregnancy to be a contraindication for such therapy due to a fear of fetal malformation. Sexual intercourse exposes women to the risk of unwanted pregnancy. It exposes many women also to another serious or more serious risk, that of sexually-transmitted infections, including HIV/AIDS. Family planning programmes with an exclusive demographic focus, cannot see the point of this important need for women.

The concept of $\mathrm{MCH}$ mainly focuses its attention on women when they are reproducing, to ensure that society gets a healthy child, but often neglects their other reproductionrelated health needs. Women's reproduction-related health needs are not limited to reproductive years of their life. The girl child, the adolescent girl, and the mature adult and older woman have health needs related to their future or past reproductive function. Nutrition of women is justified because of the needs of the fetus and the infant who needs a lactating mother. Even with the tragedy of maternal mortality, a justification put forward for investment in keeping mother alive is that their survival is critical for the survival of the children.

The concept of reproductive health has recently emerged in response to the fragmentation of the existing health services and their orientation. The broader concept of sexual and reproductive health offers a comprehensive and integrated approach to the health needs related to the sexuality and reproduction. It puts the women at the centre of the process, as subjects and not objects, as ends and not means. It recognizes respects and responds to the need of the women behind the mother.

It is a matter of great satisfaction that the present issue of Annals contains scientific articles - Surveillance and targeted action to prevent HIV/AIDS, Cancer in Women, Reproductive issues of women with epilepsy and neurocysticercosis as cause of convulsive epilepsy as disease burden in pig farming community, all contributed by very 
(iii)

experienced and learned authors, touch the above issues of women health in one way or the other.

To add as a variety there is an article on a hitherto new approach of the use of deep fascia of the skeletal muscle in cosmetic and plastic surgery. The learned contributor of this paper through a prolonged and sustained investigations has come out with very elegant path breaking research which will benefit reconstructive surgery in many ways.

Dr. Snehalata Deshmukh 